Note

\section{A Simple and Sensitive Method for the Quantitative Analysis of Chloroplast Lipids by Use of Thin Layer Chromatography and Flame Ionization Detector}

\section{Osamu Hirayama and Kohkich Morita \\ College of Agriculture, Shimane University. Matsue 690, Japan}

Received March 19, 1980

For biochemical researches, a rapid and sensitive method for the total analysis of lipid is required. Many techniques $^{1 \sim 3)}$ for the separation of lipid using TLC have been reported for this purpose. However, these still have disadvantages of tediousness and inaccuracy, because they require extraction of lipid and color development. Attempts have recently been made to combine TLC with the flame ionization detector (TLC-FID). This combination has many advantages for sensitive quantitation. Although several techniques for lipid analysis have been reported, ${ }^{4 \sim 6)}$ a satisfactory separation of plant polar lipids has not been attained.

In the present paper, we describe a new technique for the estimation of lipid and fatty acid in very small amounts of photosynthetic tissues. An improved procedure for lipid extraction from the tissues is also described here.

Lipid extraction from chloroplasts. For lipid extraction from plant tissues, the Bligh-Dyer method ${ }^{73}$ or its modified procedures have frequently been used. Bjerve et al. ${ }^{8)}$ pointed out that highly polar lipids such as sulfolipid, phosphatidylinositol and lyso-lipids were not fully extracted with the chloroform-methanol system, and recommended the use of water-saturated $n$-butanol as the solvent. This solvent, however, is disadvantageous because of its high boiling point. Thus, we devised a chloroform-isopropanol system. To $1 \mathrm{ml}$ of a chloroplast suspension containing $1 \mathrm{mg}$ chlorophyll were added $1 \mathrm{ml}$ of isopropanol and $0.6 \mathrm{ml}$ of $4 \mathrm{~N} \mathrm{H}_{2} \mathrm{SO}_{4}$. The mixture was thoroughly mixed. Chloroform $(2 \mathrm{ml})$ was added to it and the mixture was again fully mixed. After centrifugation, the mixture was separated into two layers. The upper aqueous layer was discarded, and then the bottom chloroform layer was washed twice each with $1 \mathrm{ml}$ of distilled water by centrifugations. The clear chloroform solution was taken into a pear-shaped flask, evapolated to dryness, and the remaining lipid extract was dissolved in $0.5 \mathrm{ml}$ of chloroform-methanol $(9: 1, \mathrm{v} / \mathrm{v})$. The present method is similar in the efficiency of lipid extraction to the water-saturated butanol method and superior to the Bligh-Dyer method.

Quantitative analysis of lipid from chloroplasts. Iatron Thinchrograph TFG-10 was used for the TLC-FID analysis. The lipid solution $(1 \sim 5 \mu l)$ was spotted on a silica gel sintered rod (Chromarod, S-II), and developed with chloroform-ethyl acetate-acetone-methanol-acetic acidwater $(60: 12: 15: 16: 3: 3, v / v)$. After the development, the rod was dried at $170^{\circ} \mathrm{C}$ for $10 \mathrm{~min}$ and scanned under the following conditions: flow rates of hydrogen and air, 165 and $200 \mathrm{ml}$ per $\mathrm{min}$, respectively; scanning speed, $12 \mathrm{~cm}$ per min. The amounts of each lipid component separated were estimated from the integral curve.

Figure 1 shows a typical TLC-FID chromatogram of the chloroplast lipids. All components of the polar lipids were successfully separated; they were identified by cochromatography with the authentic lipid components. The relative coefficients of each lipid component for calculation

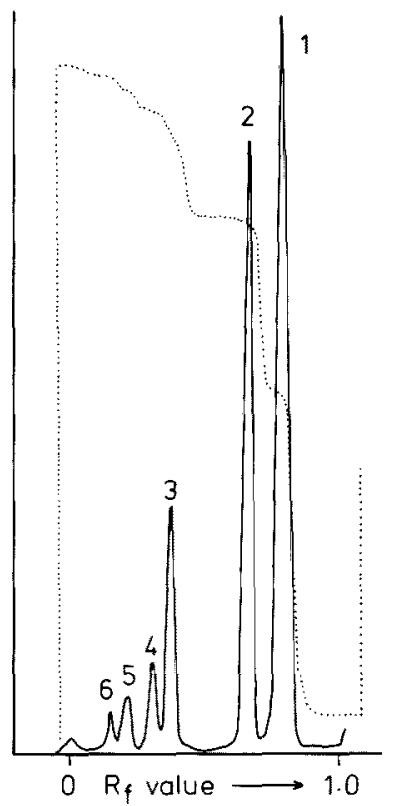

FlG. 1. TLC-FID Chromatogram of the Lipid Extract from Spinach Chloroplasts.

The sample solution was spotted on the silica gel-sintered rod (S-II), and developed with chloroform-ethyl acetate-acetone-methanol-acetic acid-water $(60: 12 \quad 15$ $16: 3: 3, v / v)$.

1 , pigments and non-polar lipids; 2, monogalactosyldiacylglycerol; 3 , digalactosyldiacylglycerol; 4 , phosphatidylglycerol; 5, sulfoquinovosyldiacylglycerol; 6, phosphatidylcholine.

The dotted line shows the integral curve 
of its content were obtained through several analyses of a known lipid mixture by the TLC-FID technique: monogalactosyldiacylglycerol, 0.88 ; digalactosyldiacylglycerol, 1.00 ; phosphatidylglycerol, 1.60 ; sulfoquinovosyldiacylglycerol, 1.60 .

Estimation of free fatty acids in chloroplasts. Estimation of free fatty acids in chloroplasts was frequently required for studying the actions of endogeneous and exogeneous lipolytic enzymes on chloroplast lipids. The lipid extract from chloroplasts, which was pheophytinized as described above, was spotted on the rod (Chromarod, S-II), and developed with $n$-hexane-ethyl ether-acetic acid $(80: 20: 1$, $\mathrm{v} / \mathrm{v}$ ). After drying at $170^{\circ} \mathrm{C}$ for $10 \mathrm{~min}$, the chromatogram was scanned. Figure 2 shows examples of the chromatograms. Pheophytin a and free fatty acids can be separated from other components, and the amounts of free fatty acids were determined by using pheophytin a as the internal standard.

Estimation of lipid in chloroplasts. Chloroplasts were washed thoroughly with distilled water by centrifugations. The chloroplast suspension $(2 \sim 5 \mu l)$ in distilled water

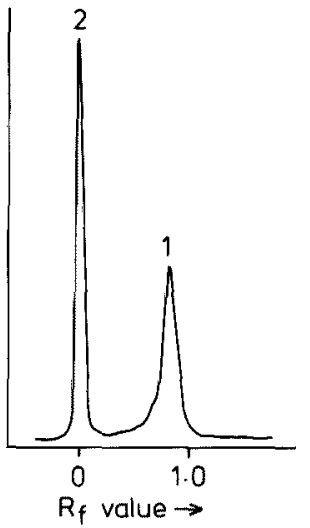

FIg. 3. TLC-FID Chromatogram for Determination of Lipid in Chloroplasts.

The washed chloroplasts in distilled water was spotted on the rod (S-1), then dried under vacuum, and developed with $n$-butanol-acetic acid-water $(60: 20: 20$, $\mathrm{v} / \mathrm{v})$.

1, lipid-soluble components; 2 , proteins.

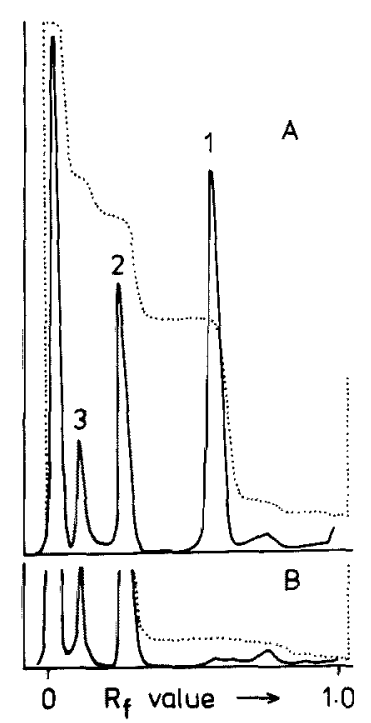

FIG. 2. TLC-FID Chromatograms for Determination of Free Fatty Acids in Chloroplasts.

The pheophytinized lipid extract from chloroplasts was spotted on the rod (S-II), and developed with $n$-hexaneethyl ether-acetic acid $(80: 20: 1, \mathrm{v} / \mathrm{v})$ as the solvent system.

1 , free fatty acids; 2 , pheophytin a; 3 , pheophytin $b$.

A, lipid extract from lipolytic enzyme-treated spinach chloroplasts; B, lipid extract from spinach chloroplasts. The dotted line shows the integral curve. containing $1 \mathrm{mg}$ chlorophyll per $\mathrm{ml}$ was spotted on the rod (Chromarod, S-I) $4.5 \mathrm{~cm}$ from its end, dried under vacuum, and developed with $n$-butanol-acetic acid-water $(60: 20: 20, \mathrm{v} / \mathrm{v})$ to $4 \mathrm{~cm}$ from the origin. After drying at $170^{\circ} \mathrm{C}$ for $30 \mathrm{~min}$, the rod was scanned as described above. Figure 3 shows one of the chromatograms, in which two peaks of the lipid-soluble components moved and the proteins remained at the origin. With the solvent system used all the lipid-soluble components containing polar lipids were extracted from chloroplasts and moved on the rod. These results were obtained by using the chloroplasts extracted to various extents with chloroform, acetone, and $n$-butanol. The coefficients for calculation of their amounts were determined from the relative responses of a given amount of lipid (chloroplast lipids), protein (bovine serum albumin), and carbohydrate (soluble starch): lipid, 1.00; protein, 1.85; carbohydrate, 4.2 . This technique makes us possible to estimate the lipid content even in very small amounts of materials.

In the present techniques of TLC-FID, the quality of silica gel-sintered rods is especially important for reproducible quantitation. Thus, it is necessary that the rods to be used are strictly selected so that all the rods show the same quantitation for the same lipid mixture. The present methods are useful for the separation and quantitation of chloroplast lipids and also applicable to other plant tissue lipids and animal lipids if some modifications are added.

\section{REFERENCES}

1) M. Kats, "Techniques of Lipidology," ed. by T. S. 
Work and E. Work, North-Holland Publishing Company, Amsterdam, 1972.

2) P. Pohl, H. Glasl and H. Wagner, J. Chromatog., 49, 488 (1970).

3) O. Hirayama and H. Matsuda, Agric. Biol. Chem., 36, 2593 (1972).

4) M. Tanaka, T. Itoh and H. Kaneko, Yukagaku, 25, 263 (1976).
5) M. Tanaka, T. Itoh and H. Kaneko, ibid., 26, 454 (1977).

6) M. Tanaka, T. Itoh and H. Kaneko, ibid., 28, 96 (1979).

7) E. G. Blight and W. J. Deyer, Can. J, Biochem. Physiol., 37, 911 (1959).

8) K. S. Bjerve, L. N. W. Daae and J. Bremer, Anal. Biochem., 58, 238 (1974). 